\title{
Factors Influencing Measurement Accuracy of Electronic Transformers
}

\author{
Qiao $\mathrm{Wu}^{1,2, *}$, Wang $\mathrm{Fu}^{3}$, Shuangshuang Zhao ${ }^{1,2,}$, Yongkun $\mathrm{Li}^{3}$, Shihai Yang, Mingming $\mathrm{Chen}^{1,2,}$, Min-rui $\mathrm{Xu}^{1,2,}$ \\ ${ }^{1}$ Electric Power Research Institute, Jiangsu Electric Power Company, Nanjing 211103, China \\ ${ }^{2}$ Key Laboratory of Electric Energy Metering, State Grid Corporation, Nanjing 210019, China \\ ${ }^{3}$ School of Electrical Engineering, Southeast University, Nanjing 210096, China
}

\begin{abstract}
With the development of smart grid in our country, as the key devices of the digital metering system, the conversion accuracy and stability of electronic transformer plays a dominant role in the whole metering system error control, the stability and reliability of the electronic transformer is related to the settlement of fair trade. But as a result of using the new sensors and electronic components, the electronic transformer can be easily affected by the outside factors, causing the low stability and reliability during its long-term operation. The paper introduces the classification of the electronic transformer, and analyses working principle and influence factors of typical electronic voltage transformers.
\end{abstract}

\section{Introduction}

The digital metering system is considered an integral part of the intelligent substation, and the electronic transformer is the key device of the digital metering system, the stability and reliability of the electronic transformer is related to the settlement of fair trade. But as a result of using the new sensors and electronic components, the electronic transformer can be easily affected by the outside factors, such as electromagnetism environment, temperature and moisture, vibration etc,causing the low stability and reliability during its long-term operation ${ }^{[1-5]}$.

The paper introduces the classification of the electronic transformer, and classifies the electronic transformer into two major categories of electronic current transformer and electronic voltage transformer, according to whether the high side sensor system needs power supply or nor, electronic current transformer and electronic voltage transformer are divided into active type and passive type ${ }^{[6]}$. And introduce the working principle of several kinds of typical electronic transformers, mainly analyzing various kinds of influence factors of electronic transformer. ${ }^{[7-9]}$

\section{Classification of electronic transformers}

Many kinds of electronic transformers have been widely used in intelligent substation pilot project, and a variety of classifications are existed. The paper divides the electronic current transformer and electronic voltage transformer into active type and passive type. As shown in figure 1.

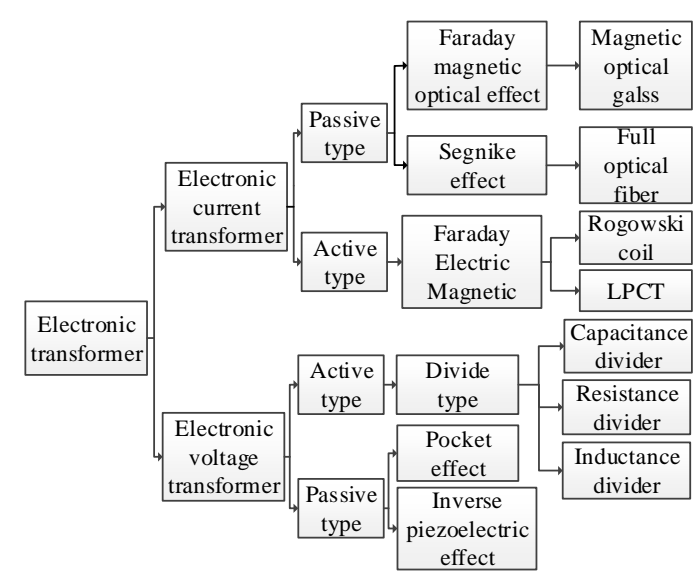

Figure 1. Electronic transformer classification

\section{Electronic current transformer}

\subsection{Magnetic optical glass current transformer}

\subsubsection{Principle of magnetic optical glass current transformer}

Passive electronic current transformer is also called optical current transformer. Optical current transformer based on the Faraday magnetic optical effect is mainly researched at present, and the schematic of its sensor is shown in figure 2.

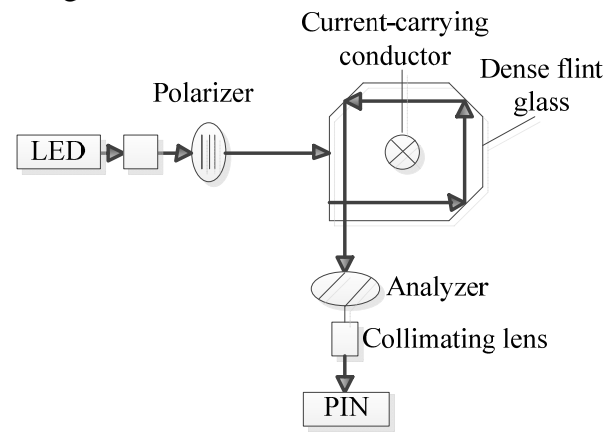

Figure 2. Principle diagram of passive electronic current sensor 
The LED light turns to be a linearly polarized light through the polarizer, and revolves after surrounding the dense flint glass a circle. According to the Faraday magnetic optical effect ${ }^{[10]}$ and Ampere circuit law, linearly polarized light rotation angle $\theta$ and the flow of current $i$ in conductor have the following relationship:

$$
\theta=V \int H d l=V \int H d l=V i
$$

Where: $V$ is Verdet constant.

Linearly polarized light rotation angle $\theta$ is proportional to the current $i$ being measured, and the analyzer converts the change of the angle to the change of the output light intensity, after photovoltaic conversion and signal processing, the current can be measured.

\subsubsection{Factors affecting magnetic optical glass current transformer}

(1) Temperature effect.The change of temperature leads to the change to Verdet constant and the wave length of the LED light, which also result in the change of Verdet constant.

(2) Optical material effect. Stress can cause birefringence when assembled, and the light path system is quite complicated when using optical method to weaken the interference of birefringence.

(3) Vibration effect. Due to the photoelastic effect of the optical material, the vibration of the periodic can cause the sensor periodic change of birefringence, which affects the output stability of the system.

\subsection{Rogowski coil current transformer}

\subsubsection{Principle of Rogowski coil current transformer}

Rogowski coil current transformer using the Rogowski coil measures the magnetic field causing by the current in the conductor to calculate the current. Rogowski coil is a tightly solenoid coil winding on the non-magnetic skeleton. When the coil geometry size is uniform, the induction electric potential is shown as follow.

$$
e(t)=-\frac{d \phi}{d t}=-\frac{u_{0} N h}{2 \pi} \ln \frac{r_{0}}{r_{i}} \cdot \frac{d I}{d t}
$$

Where: $r_{0}$ is outer diameter and $r_{\mathrm{i}}$ is inner diameter.

Considering the distribution parameter, the output voltage of the Rogowski coil can be calculated by the formula below.

$$
\begin{aligned}
U_{0} & =E(t) \cdot \frac{1 / j \omega C_{0}}{j \omega L+R_{0}+1 / j \omega C_{0}} \\
& =E(t) \cdot \frac{1-\omega^{2} L C_{0}-j \omega R_{0} C_{0}}{\left(1-\omega^{2} L C_{0}\right)^{2}+\left(\omega R_{0} C_{0}\right)^{2}}
\end{aligned}
$$

\subsubsection{Factors affecting Rogowski coil current transformer}

(1) Geometry size effect. The Rogowski coil geometry size includes inner diameter, outer diameter, height and thread pitch, the output voltage can be influenced by any change of parameters above.

(2) Deviate from circle center effect. Primary side conductor deviating from circle center, the output voltage of the coil will change.

(3) Magnetic-field interference effect. The interference potential of magnetic-field interference will be generated in the envelope of the Rogowski coil. Because the area of the equivalent envelope is larger than the cross-sectional area of the coil, if the processing is not good, the high accuracy of the current transformer will be affected a lot.

\section{Electronic Voltage Transformer}

\subsection{Passive electronic voltage transformer}

\subsubsection{Principle of passive electronic voltage transformer}

Passive electronic voltage transformer is also often referred to as optical voltage transformer and the measuring principle of optical voltage transformer can be roughly divided into two types based on Pockels effect and converse piezoelectric effect. The optical voltage transformer based on Pockels effect is being investigated extensively, as shown in figure 3

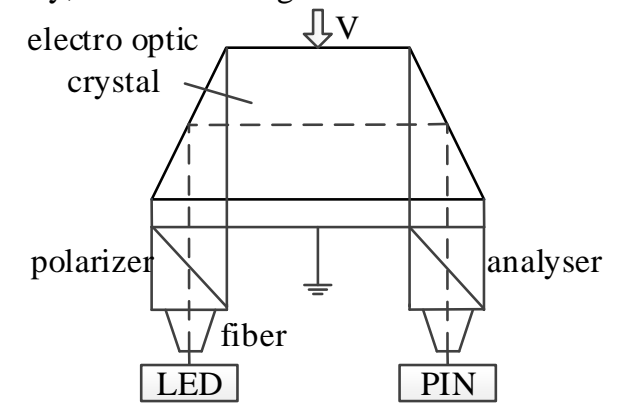

Figure 3. Schematic diagram of optical voltage transformer based on Pockels effect

The light emitted from the LED becomes a linearly polarized light through the polarizer. Under the applied voltage, the linearly polarized light occurs birefringence by the electro optic crystal (such as BGO). The phase difference $\delta$ of the two light beams after birefringence and the applied voltage $V$ have the following relations:

$$
\begin{gathered}
\delta=\frac{2 \pi}{\lambda} n_{0}^{3} \gamma_{41} \frac{l}{d} V=\frac{\pi}{V_{\pi}} V \\
V_{\pi}=\frac{\lambda}{2 n_{0}^{3} \gamma_{41}} \frac{d}{l}
\end{gathered}
$$

Where: $n_{0}$ is the refractive index of BGO, $\gamma_{41}$ is the electro-optic coefficient of BGO, $l$ is the optical path length of BGO, $d$ is the thickness of BGO in the direction of the applied voltage, $\lambda$ is wavelength of incident light, $V_{\pi}$ is the half-wave voltage of crystal.

The phase difference $\delta$ is proportional to the applied voltage $V$. The change of phase difference $\delta$ is converted to the variation in output light intensity by using the 
analyzer, and the measured voltage can be obtained by photoelectric conversion and corresponding signal processing.

\subsubsection{Factors affecting passive electronic voltage transformer}

(1) Temperature effect. Optical elements in optical voltage transformers are bonded by optical adhesive, and the change of temperature can cause the change of the optical path system. The optical path system has a long delay time with the change of temperature, and the repeatability is poor.

(2) Effect of electro optic crystal. BGO crystal is the most widely used in the optical electronic crystal, which contains a number of precipitates, which can cause birefringence and bring additional phase delay. At the same time, crystal can exist the effect of elastic-optic effect and thermo-optic effect, absorption and dispersion, optical activity, which will cause the change of refractive index of the crystal.

\subsection{Active electronic voltage transformer}

\subsubsection{Principle of active electronic voltage transformer}

Active electronic voltage transformer converts the measured voltage to low voltage signal by capacitive voltage divider, resistance divider or series circuit of capacitance and resistance. After a series of processing, the small signal is converted into optical signal by $E / O$, and transmitted to the low voltage side by the fiber, and also transmitted to the secondary protection and control equipment after the data processing of the low voltage side. The paper introduces the electronic voltage transformer based on capacitive voltage divider.

The ideal equivalent model of capacitor voltage divider is shown in figure 4 . Capacitive voltage divider is composed of the high voltage arm capacitor $C_{1}$ and the low voltage arm capacitor $\mathrm{C}_{2}$, which converts the voltage of high-voltage end to the voltage of low-voltage terminal (amplitude is usually only a few volts) and A/D transform, then fiber transmits the signal by electro-optic conversion and couples to signal handling unit for photoelectric conversion, and outputs digital signal through microcomputer system processing or output analog signal by $\mathrm{D} / \mathrm{A}$ conversion.

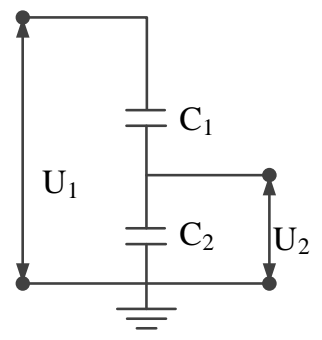

Figure 4. The ideal equivalent model of capacitor voltage divider

The ideal voltage division formulas in figure 4 can be expressed as:

$$
U_{2}=\frac{C_{1}}{C_{1}+C_{2}} U_{1}=k U_{1}
$$

Where: $k$ represents the ideal partial pressure ratio of active electronic voltage transformer;

When $C_{1}$ and $C_{2}$ are stable, the ideal partial pressure ratio is stable.

\subsubsection{Factors affecting active electronic voltage transformer}

(1) Influence of stray capacitance on the partial pressure ratio. Stray capacitance exists in the practical electronic voltage transformer based on the principle of capacitive voltage divider. Stray capacitance can change the potential distribution of divider interior. And a part of the displacement current shunts through the stray capacitance, thereby affecting the output of secondary voltage, which causes the change of the partial pressure ratio.

(2) Influence of proximity effect on the partial pressure ratio. The coupling capacitance exists between capacitor cells of electronic voltage transformer based on the principle of capacitive voltage divider of different phases, which will cause some displacement current of electronic voltage transformer flowing into the electronic voltage transformer of the adjacent phase through the coupling capacitor, and the voltage drop is formed on the distributed capacitance of main capacitor at low-voltage terminal, thereby affecting the output voltage of voltage divider of electronic voltage transformer of the adjacent phase.

\section{Conclusion}

In this paper, electronic transformers are classified as electronic current transformers and electronic voltage transformers. Then several typical electronic transformers are introduced and analyzed about the influence factors, including passive electronic current transformers based on the principle of Faraday effect and active electronic current transformer based on Rogowski coil, and passive electronic voltage transformer based on the principle of Pockels effect and the active electronic voltage transformer based on capacitive voltage divider.

Electronic transformer has the advantages of antielectromagnetic interference, unsaturated, large measurement range, small volume, light weight and so on, which can meet the requirements of the development of modern power system. Through the analysis of the working principle and the influence factors of electronic transformer, the paper finds that sensing head of the high voltage side of electronic transformer is in the high voltage and high current. The interference source is mainly derived from the surrounding electrical equipment, as well as the influence of the non-electromagnetic factors including temperature, vibration and so on. In the future, the various influence factors and solutions to solve the problem of the stability and the reliability of electronic transformer need further study. 


\section{Acknowledgements}

This work was supported by the Science and Technology Foundation of State Grid Corporation.

\section{References}

[1] J. Li, Y. Yang, W. Yu, G. Zhang. Review of electric energy metering system. Power System Protection and Control, 11, 130-134 (2009).

[2] J. Li, Y. Zheng, S. Gu, L. Xu. Application of Electronic Instrument Transformer in Digital Substation. Automation of Electric Power Systems. 7, 94-98 (2007).

[3] P. Wang, C. Luo, G. Zhang. Electronic Current Transformer Based on Low-power Current Transformer. Automation of Electric Power Systems, 4, 98-101 (2006).

[4] H. Wang, G. Zhang, X. Cai, Z. Guo. Development of Precise Capacitive Voltage Divider for Optical Voltage Transformer. Automation of Electric Power Systems, 8, 72-87 (2009).

[5] J. Zou, Y. Liu, C. Wang, L. Xu. Development of study of optical voltage transducer used in power system. Automation of Electric Power Systems. 9, 64-67 (2001).

[6] J. Shu. Discussion on the Electronic Transformer and Its Application. Mechanical \& Electrical Engineering Technology, 2, 65-67 (2013).

[7] P. Wang, G. Zhang, L. Li, X. Zhu. Error analysis of electronic instrument transformers. J Tsinghua Univ (Sci \& Tech), 7, 1005-1008 (2007).

[8] J. Huang, J. Wang. Key technology of optical current transformer. Electric Power Automation Equipment, 12, 94-97 (2009).

[9] Z. Tian, S. Luo, J. Yang. Rogowski coil electronic current transducer applied to Protection and monitoring of GIS. Electric Power, 10, 53-56 (2003).

[10] A. Chen, Q. Yue, Y. Feng, C. Yan. Temperature performance optimization of FOCT. Electric Power Automation Equipment, 1, 142-145 (2011). 